\title{
Anti-inflammatory effects of oxymatrine on rheumatoid arthritis in rats via regulating the imbalance between Treg and Th17 cells
}

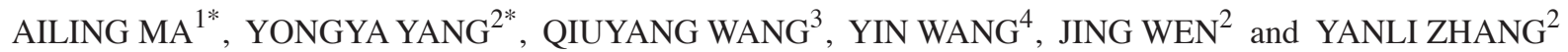 \\ ${ }^{1}$ Department of Pathology, The General Hospital of Ningxia Medical University; ${ }^{2}$ Department of Pathogen Biology \\ and Immunology, School of Basic Medical Sciences, Ningxia Medical University, Yinchuan, Ningxia 750004; \\ ${ }^{3}$ Department of Laboratory, the People's Hospital of Yan'an, Yan'an, Shanxi $71600 ;{ }^{4}$ Ningxia Key Laboratory of \\ Cerebrocranial Diseases, Ningxia Medical University, Yinchuan, Ningxia 750004, P.R. China
}

Received March 12, 2016; Accepted February 24, 2017

DOI: $10.3892 / \mathrm{mmr} .2017 .6484$

\begin{abstract}
Oxymatrine(OMT), a monosomic alkaloid extracted from the Chinese herb, Sophora flavescens Ait, has long been used as a traditional Chinese medicine for the treatment of inflammatory diseases. The aim of the present study was to investigate the potential anti-inflammatory effect of OMT, and its modulation on imbalance between regulatory $\mathrm{T}$ (Treg) cells and T helper (Th) 17 cells in rats with collagen-induced arthritis (CIA). Sprague-Dawley rats were immunized with type II collagen and following a second collagen immunization, the rats were treated with OMT or dexamethasone (DXM) intraperitoneally once a day for 43 days. Paw swelling, arthritic score and joint histopathology were evaluated. The Treg/Th17-mediated autoreactive response was assessed by determining serum levels of inflammatory response cytokines, including tumor necrosis factor (TNF)- $\alpha$ and interleukin (IL)-17, using an enzyme-linked immunosorbent assay. The mRNA levels of forkhead box P3 (FOXP3) and retinoic acid-related orphan receptor (ROR) $\gamma \mathrm{t}$ in spleen cells stimulated with type II collagen were determined using reverse transcription-quantitative polymerase chain reaction analysis. In addition, the protein expression levels of FOXP3 and ROR $\gamma \mathrm{t}$ were measured using western blot analysis. The results showed that OMT treatment significantly reduced the severity of CIA, markedly abrogating paw swelling, arthritic scores and synovial hyperplasia, and the increased loss in body weight. OMT significantly reduced the production of TNF- $\alpha$ and IL-17A, upregulated FOXP3 and downregulated ROR $\gamma \mathrm{t}$ in
\end{abstract}

Correspondence to: Professor Yanli Zhang, Department of Pathogen Biology and Immunology, School of Basic Medical Sciences, Ningxia Medical University, 1160 Shengli Street, Yinchuan, Ningxia 750004, P.R. China

E-mail:wi_ntersweet@outlook.com

*Contributed equally

Key words: inflammation collagen-induced arthritis, oxymatrine, regulatory $\mathrm{T}$ cell, helper $\mathrm{T} 17$ cell rats with CIA. In conclusion, the present study demonstrated that OMT exhibited a protective effect on rheumatoid arthritis (RA) through the inhibition of inflammation and regulation of Treg/Th17 in the CIA rats, suggesting that OMT may be used as an immune suppressive and cartilage protective medicine in human RA.

\section{Introduction}

Rheumatoid arthritis (RA), a common and systemic autoimmune disease of unknown etiology, leads to chronic progressive and aggressive inflammation in the synovial joints, with subsequent destruction of cartilage and erosion of bone in the affected joint, which causes severe disability and increased mortality rates $(1,2)$. In RA, inflammatory cells, including natural killer cells, T and B lymphocytes and neutrophils, infiltrate the synovial membrane, contributing to cartilage and bone degradation (3). Among the proinflammatory cytokines, tumor necrosis factor- $\alpha$ (TNF- $\alpha$ ) and interleukin 17A (IL-17A) are expressed at high levels in the rheumatoid joint and are important in the mechanisms underlying the inflammatory response $(4,5)$. These cytokines, produced by $\mathrm{CD}^{+} \mathrm{T}$ helper (Th) cells, and $\mathrm{CD}^{+} \mathrm{T}$ cells are important in the inflammatory process via their cytolytic activities, and the production of pro- and anti-inflammatory cytokines, which regulate immune responses. $\mathrm{CD}^{+} \mathrm{T}$ cells can differentiate into Th1, Th2, Th9, Th17 or regulatory $\mathrm{T}$ (Treg) cells, depending on the cytokine secretion and expression of specific transcription factors (6). Th17 cells were identified in 2005 based on their ability to produce IL-17A $(7,8)$. CD4 ${ }^{+}$lymphopenia in patients is usually caused by diseases, including RA (9-11). Among the identified T cell subsets, Th17 and Treg cells have gained increasing scientific interest and have been extensively investigated in several autoimmune/inflammatory disorders (12-15). Pathogenic Th17 cells are key in the development of RA, which mediate pannus growth, osteoclastogenesis and synovial neoangiogenesis. By contrast, Treg cells are a T cell subset functioning to suppress autoreactive lymphocytes. The imbalance between Th17 cells and Treg cells has been identified as a crucial event in the pathogenesis of RA $(16,17)$.

According to previous reports, RA affects $\sim 1 \%$ of the adult population in developed countries $(18,19)$. Treatment 
for RA has been categorized into the use of disease-modifying anti-rheumatic drugs (DMARDs) and non-steroidal anti-inflammatory drugs (NSAIDs). Traditional NSAIDs, including ibuprofen and diclofenac, inhibit cyclooxygenase (COX) I and COX II (20). They reduce pain and swelling in RA, enhance recovery, and promote mobility and physical activity. However, NSAIDs do not slow the progression of the disease and may have adverse effects (21). The use of NSAIDs is associated with cardiovascular risk factors due to their effect in increasing systolic blood pressure, particularly in high-risk patients with diabetes, hypertension or heart disease $(22,23)$. Conventional DMARDs are immunosuppressive agents, of which methotrexate (MTX) is the most commonly used, and remain the cornerstone of RA treatment. In previous years, treatment strategies and the use of DMARDs have changed. DMARDs retard or halt disease progression, or delay disease onset; 'tight control' and 'treat-to-target' are the presently used paradigms (24). However, DMARDS do not suppress the progression of clinical disability $(25,26)$. Novel therapeutic approaches are required to identify a therapeutic target for remission or low disease activity, which can reduce the inflammatory and autoimmune components in RA, promote restoration of immune tolerance, slow cartilage destruction and reduce the treatment time for RA (27).

Oxymatrine (OMT), a type of monosomic alkaloid extracted from the dried roots of the traditional Chinese herb, Sophora flavescens Ait. (Kushen) or Sophora alopecuroides (Kudouzi), has a tetracyclic quinolizine structure, its molecular formula is $\mathrm{C}_{15} \mathrm{H}_{24} \mathrm{~N}_{2} \mathrm{O}$. OMT possesses potent anti-inflammatory, immunoregulatory, antivirus, anticancer, antifibrotic and cardiovascular-protective activities (28-32). Previously, OMT studies have focused predominantly on its therapeutic effect against other inflammatory diseases, certain types of tumor and hepatitis (33-35). There have been few reports on the effect of OMT on autoimmune diseases, including RA. The aim of the present study was to evaluate the effect and mechanism of OMT treatment on RA.

\section{Materials and methods}

Drugs and chemicals. OMT was purchased from Ningxia Bauhinia Pharmacy Co., Ltd. (Ningxia, China). OMT 100, 50 and $25 \mathrm{mg} / \mathrm{kg}$ (dissolved in normal saline) was administrated via intraperitoneal injection (i.p.). Immunization grade bovine type II collagen and complete Freund's adjuvant were purchased from Chondrex, Inc. (Redmond, WA, USA). Enzyme-linked immunosorbent assay (ELISA) kits for IL-17 and TNF- $\alpha$, and mouse monoclonal antibodies against FOXP3 (ab22510), ROR $\gamma$ t (ab41942) and $\beta$-actin (ab8226) were purchased from Abcam (Cambridge, MA, USA). TRIzol was obtained from Gibco; Thermo Fisher Scientific, Inc. (Waltham, MA, USA). A reverse transcription kit was purchased from TransGen Biotech, Inc. (Beijing, China). Reverse transcription-quantitative polymerase chain reaction (RT-qPCR) analysis was performed using GoTaq ${ }^{\circledR}$ qPCR master mix (Promega Corporation, Madison, WI, USA). BCA and enhanced chemiluminescence (ECL) kits were from Pierce, Thermo Fishers Scientific, Inc.

Animals. Male Sprague-Dawley (SD) rats (8 weeks old; 180-220 g) were obtained from the Experimental Animal
Center, Ningxia Medical University (Ningxia, China). They were housed in multilayer laminar flow racks under a controlled environment $\left(20-25^{\circ} \mathrm{C}\right.$ and $12 \mathrm{~h}$ light:dark cycle) with free access to food and water. The present study was performed according to the Guiding Principles for the Care and Use of Laboratory Animals (36) and all procedures were approved by the Animal Care and Use Committee of Ningxia Medical University.

Half lethal dose $\left(L D_{50}\right)$ assay. The $\mathrm{LD}_{50}$ of OMT was measured using a sequential method with five dose levels according to body weight, with a single i.p. injection. The mortality rates of the rats were monitored during the 14 days follow treatment.

Induction of collagen-induced arthritis (CIA) and OMT treatment. The Male SD rats [Permit no. SCXK (Ning) 2011-0001] were randomly divided into six groups (10 rats/group) prior to the onset of arthritis: Normal control group, positive control group treated with dexamethasone (DXM; $2 \mathrm{mg} / \mathrm{kg}$, twice a week), CIA model group, OMT high-dose group (100 mg/kg, once daily), middle-dose group (50 mg/kg, once daily) and low-dose group (25 mg/kg, once daily). The 50 male SD rats, excluding those in the normal control group (10 rats) were administered with a subcutaneous injection of $0.1 \mathrm{ml}$ bovine type II collagen emulsified in complete Freund's adjuvant (1:1, v/v) into the right hind metatarsal footpad. After 1 week, the rats were administered with a booster subcutaneous injection of $0.1 \mathrm{ml}$ bovine CII in incomplete Freund's adjuvant $(1: 1, \mathrm{v} / \mathrm{v})$ into the left hind metatarsal footpad. The control rats were treated in the same manner but without the CII antigen. Between days 1 and 35 following the second immunization, the rats in the OMT-treated group were administered with OMT at concentrations of 100,50 or $25 \mathrm{mg} / \mathrm{kg}$ i.p. The rats in the control group and the CIA model group were administered with $100 \mathrm{mg} / \mathrm{kg}$ saline i.p., and DXM $(2 \mathrm{mg} / \mathrm{kg}$ ) was used as a reference drug, administered (i.p.) at the same time. The gradual onset of arthritis usually starts $\sim 10$ days following primary immunization.

\section{Evaluation of CIA}

Hindpaw swelling. The volume of the hindpaw swelling was measured with vernier calipers once every 4 days for 6 weeks. Hindpaw swelling $\left(\mathrm{mm}^{2}\right)=$ left hindpaw swelling $(\mathrm{mm}) \mathrm{x}$ left hind ankle swelling (mm).

Arthritis score. The SD rats were assessed every 4 days for the progression of CIA between days 1 and 35 following secondary immunization. Each paw was examined and graded for severity of erythema, swelling and scleroma, and the four scores were combined, resulting in a maximum possible score of 16 per mouse; the maximum arthritic score per rat was set at 8 (4 points for two hindpaws). The score was calculated using a five-point scale: 0 , no signs of arthritis; 1 , signs involving the ankle/wrist; 2, signs involving the ankle+tarsal of the hindpaw and/or wrist+carpals of the forepaw; 3 , signs extending to the metatarsals or metacarpals; 4 , severe disease involving the entire hindpaw or forepaw. The examination was performed by two independent observers who remained blinded to the treatment groups. 


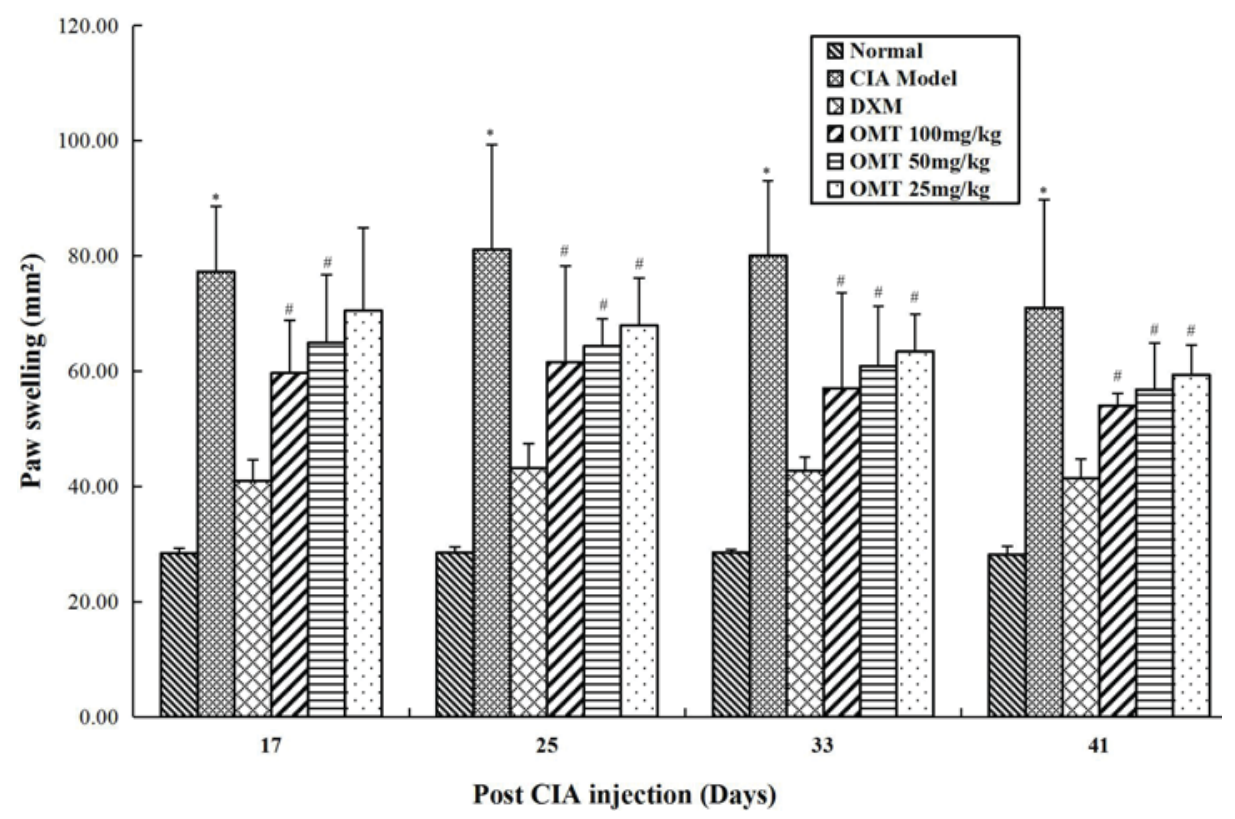

Figure 1. Effects of OMT on paw swelling in rats. Treatment with OMT (100,50 and $25 \mathrm{mg} / \mathrm{kg} / \mathrm{day}$ i.p.) between days 8 and 35 relieved paw swelling, compared with the arthritic group on days 18-24 following secondary immunization. Data are presented as the mean \pm standard deviation ( $\mathrm{n}=10 /$ group). ${ }^{*} \mathrm{P}<0.05$, compared with the normal group; ${ }^{\#} \mathrm{P}<0.05$, compared with the CIA model group. OMT, oxymatrine; CIA, collagen-induced arthritis; DXM, dexamethasone.

Histological analysis of knee joints. The SD rats were sacrificed via anesthesia and serum was collected on day 35 following second immunization. The knee joints were dissected, fixed in $4 \%$ paraformaldehyde solution for $24 \mathrm{~h}$, decalcified in $10 \%$ ethylene diamine tetraacetate for 30 days, with the solution renewed once a week, and then embedded in paraffin. Standard frontal sections of $3 \mu \mathrm{m}$ were prepared and stained with hematoxylin and eosin (H\&E). The synovial tissue sections were observed using light microscopy (CX23; Olympus Corporation, Tokyo, Japan) and evaluated in a blinded-manner.

Measurement of serum levels of IL-17A and TNF- $\alpha$. The serum levels of IL-17A and TNF- $\alpha$ were quantified using ELISA according to the manufacturer's protocol (Abcam). For measurements of IL-17A and TNF- $\alpha$, the SD rats were anesthetized on the final day of the experiment and serum was drawn from the heart. The reaction product was quantified using a microplate reader (Bio-Rad Laboratories, Inc., Hercules, CA, USA) at $450 \mathrm{~nm}$. All samples were analyzed in duplicates using the average optical density values to calculate concentrations.

Spleen lymphocyte preparation. The spleens were removed immediately following sacrifice and placed in PBS. The spleens were then mechanically disrupted through a wire mesh strainer with the end of a $10 \mathrm{ml}$ plastic syringe plunger. The spleen lymphocytes preparations were filtered through lens tissue to remove debris and the cells were collected by centrifugation (at $500 \mathrm{xg}$ for $5 \mathrm{~min}$ at $4^{\circ} \mathrm{C}$ ). Finally, the cells were resuspended, dispersed in trypsin and counted using a hemocytometer.

RNA extraction and RT-qPCR analysis. Total RNA was extracted from the spleen lymphocytes using TRIzol. The purity and concentration of RNA was determined using spectrophotometry at 260 and $280 \mathrm{~nm}$. Complementary DNA was synthesized using a reverse transcription kit. The RT-qPCR mixture was denatured at $95^{\circ} \mathrm{C}$ for 2 min followed by 50 cycles of amplification including denaturing at $95^{\circ} \mathrm{C}$ for $15 \mathrm{sec}$, annealing at $60^{\circ} \mathrm{C}$ for $15 \mathrm{sec}$ and extension at $72^{\circ} \mathrm{C}$ for $45 \mathrm{sec}$. The total reaction volume was $20 \mu \mathrm{l}$ with $3 \mu \mathrm{l}$ cDNA, $10 \mu 1$ ROX qPCR Master (2X), $0.5 \mu 1$ primers individually

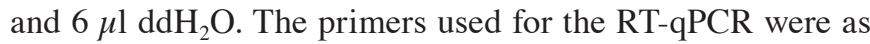
follows: ROR $\gamma$ t, sense 5'-TCTGGAAGCTGTGGGATAGA-3' and antisense 5'-GAG-GAGCCTGTGGAGAAATAC-3'; FOXP3, sense 5'-GGCCCTTCTCCAGGACAGA-3' and antisense 5'-GCTGATCATGGCTGGGTTGT-3'; $\beta$-actin, sense 5'-CCTCATGCCATCCTGCGTCT-3' and antisense 5'-GCC ACAAGGATTCCATACCCA-3'. Relative gene expression levels were determined as described previously (37). The results were normalized to the expression of the housekeeping gene, $\beta$-actin. Data shown are representative of three independent experiments. Data analysis was performed using the $2^{-\Delta \Delta C q}$ method (38).

Western blot analysis. Spleen lymphocytes were washed with PBS three times and lysed with RIPA buffer. The protein concentration was determined using a BCA kit according to the manufacturer's protocol. Protein $(20 \mu \mathrm{g})$ was separated by $10 \%$ SDS-PAGE and then transferred onto a PVDF membrane. The membranes were blocked with 5\% dried milk and incubated with primary antibody against FOXP3 $(1: 1,000)$ and $\operatorname{ROR} \gamma t(1: 2,000)$ in TBST overnight at $4^{\circ} \mathrm{C}$. Following rinsing in milk-TBST, the blots were incubated with horseradish peroxidase-conjugated secondary antibody at room temperature for $1 \mathrm{~h}$. The expression levels of FOXP3 and ROR $\gamma \mathrm{t}$ were detected using the ECL detection system and X-ray films.

Statistical analyses. Data are presented as the mean \pm standard deviation. Data were analyzed using Student's t-test and 


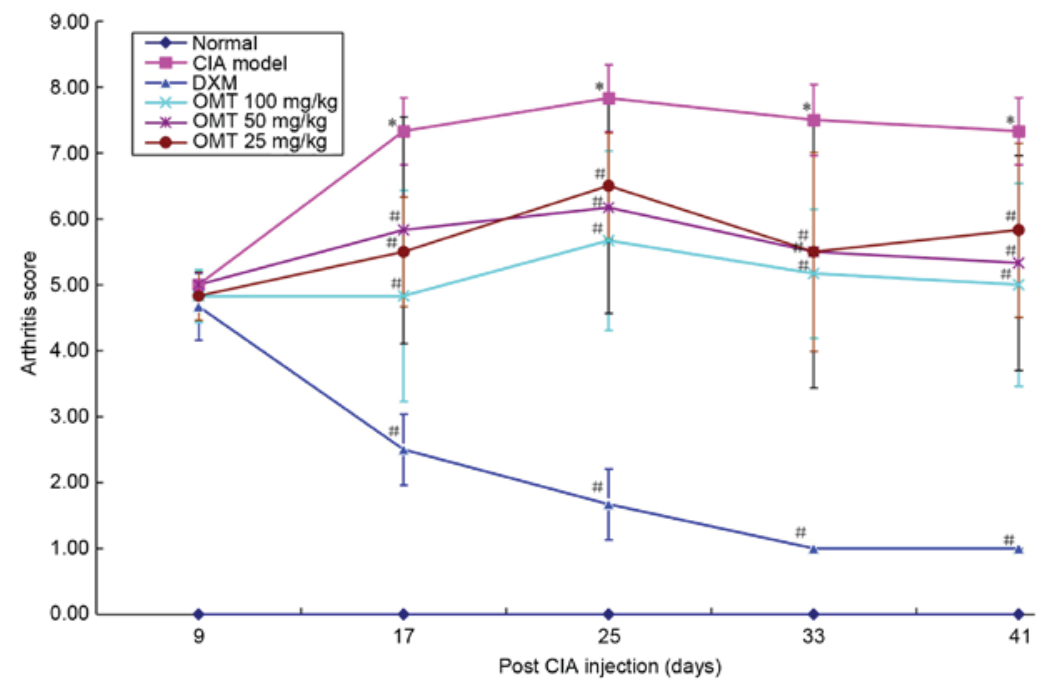

Figure 2. Effects of OMT on arthritis in rats, as assessed using arthritic scores. Treatment with OMT (100, 50 and $25 \mathrm{mg} / \mathrm{kg} / \mathrm{day}$ i.p.) between days 8 and 35 significantly decreased the arthritic score and suppressed peak arthritic severity, compared with the CIA model group between days 17 and 41 . Data are presented as the mean \pm standard deviation ( $\mathrm{n}=10$ /group). ${ }^{\mathrm{P}}<0.05$, compared with the normal group; ${ }^{\mathrm{P}} \mathrm{P}<0.05$, compared with the CIA model group. OMT, oxymatrine; CIA, collagen-induced arthritis; DXM, dexamethasone.
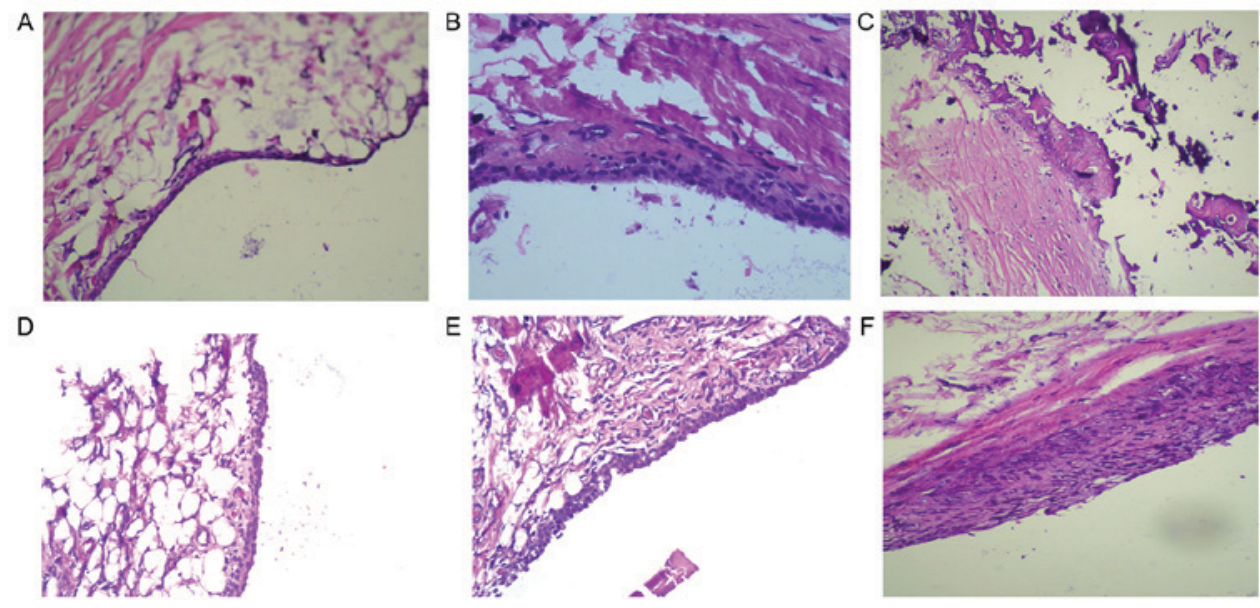

Figure 3. Effects of OMT on histopathological changes in the paw section of CIA rats, using hematoxylin and eosin staining. (A) Normal group rats. (B) CIA model group rats showed marked infiltration of inflammatory cells and synovial hyperplasia. (C) CIA rats treated with dexamethasone. (D) $100 \mathrm{mg} / \mathrm{kg}$ OMT-treated CIA rats. (E) $50 \mathrm{mg} / \mathrm{kg}$ OMT-treated CIA rats. (F) $25 \mathrm{mg} / \mathrm{kg}$ OMT-treated CIA rats. Treatment with OTM led to relief of synovial hyperplasia and inflammatory cell infiltration. (Magnification, x400). OMT, oxymatrine; CIA, collagen-induced arthritis.

one-way analysis of variance. Statistical significance in the comparison of means of different groups was calculated using LSD-t analyses with SPSS version 13.0 (SPSS, Inc., Chicago, IL, USA). $\mathrm{P}<0.05$ was considered to indicate a statistically significant difference between data sets.

\section{Results}

Toxicity of OMT. To evaluate the toxicity of the i.p. injection of OMT, the present study determined its $\mathrm{LD}_{50}$ in rats. The $\mathrm{LD}_{50}$ of OMT was $898.22 \mathrm{mg} / \mathrm{kg}$ (95\% confidence interval, 832.46-963.98).

OMT attenuates collagen-induced arthritis in SD rats. There was a significant increase in hindpaw volumes in all CIA groups, compared with those in the normal control group. Hindpaw swelling involved tarsal, distal with ankle and interphalangeal inflammation. The onset of arthritis was induced in all immunized rats in $24 \mathrm{~h}$. The first manifestation of CIA was erythema of one or more ankle joints, followed by involvement of the metatarsal and interphalangeal joints. The development of erythema reached a peak on day 9, following which there was a mild decrease in the signs of inflammation between days 10 and 16. However, on days 17-18, hindpaw swelling was followed by the rapid reappearance of inflammation. The rats treated with 100 and $50 \mathrm{mg} / \mathrm{kg}$ OMT showed significant reduction in paw edema volume, compared with that in the model group. Treatment with OMT (100, 50 and $25 \mathrm{mg} / \mathrm{kg} / \mathrm{day}$, days 8-35) relieved the right hindpaw swelling and inhibited the progression of polyarthritis between days 18 and 24 following secondary immunization (Fig. 1). The same efficacy of DXM (2 mg/kg, twice/week, ig, days 8-35) were observed.

To examine the effects of OMT on arthritic progression in rats with CIA, the development of arthritis was evaluated by 

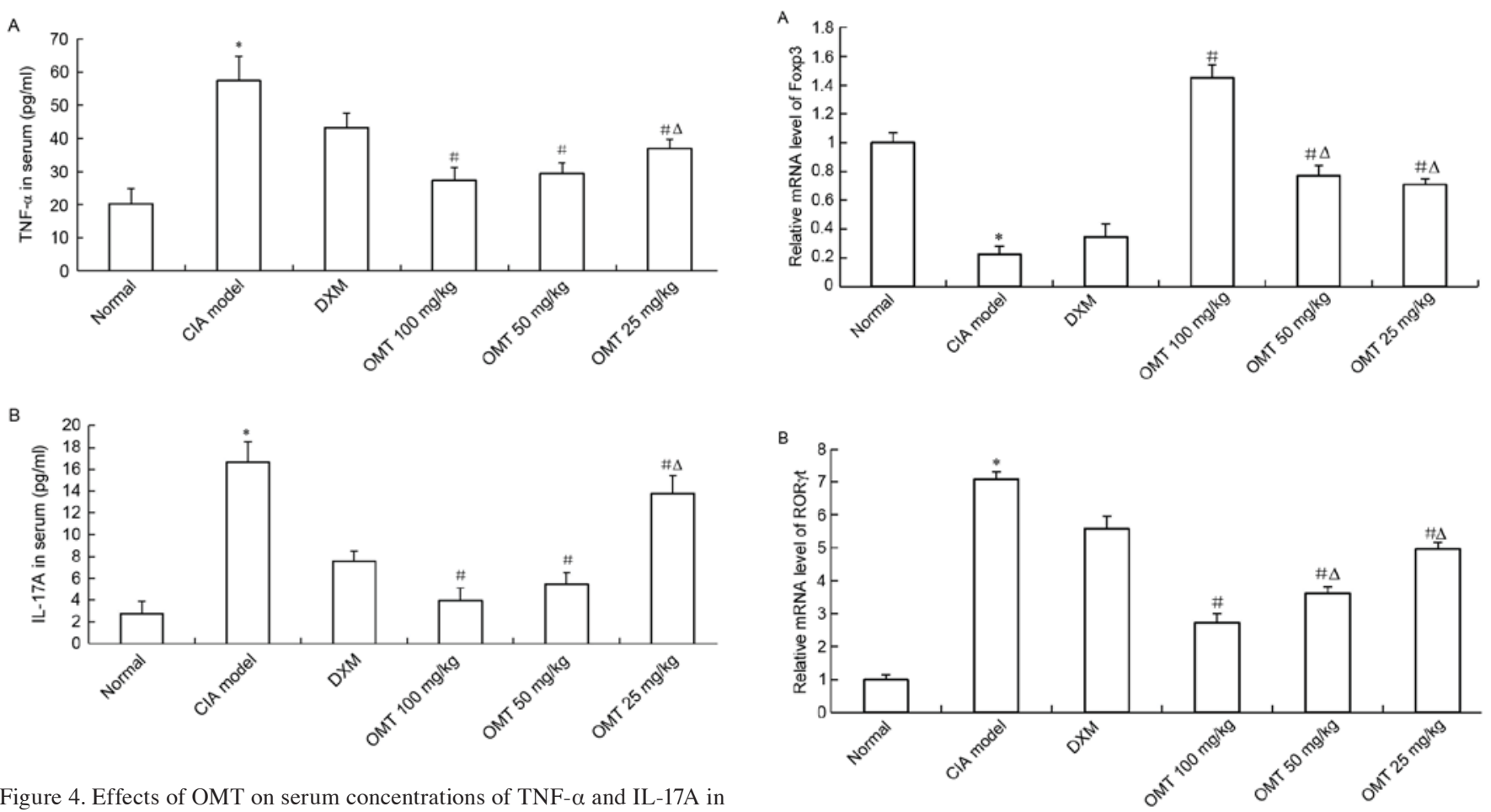

Figure 4. Effects of OMT on serum concentrations of TNF- $\alpha$ and IL-17A in CIA rats, measured using enzyme-linked immunosorbant assays. (A) TNF- $\alpha$; (B) IL-17A. Levels of TNF- $\alpha$ and IL-17A were markedly reduced by OMT concentrations of 100,50 and $25 \mathrm{mg} / \mathrm{kg}$, in a dose-dependent manner. Results are representative of three independent experiments. ${ }^{*} \mathrm{P}<0.05$, compared with the normal group; ${ }^{\#} \mathrm{P}<0.05$, compared with the CIA model group; ${ }^{\mathrm{P}} \mathrm{P}<0.05$, compared with the $100 \mathrm{mg} / \mathrm{kg}$ group. OMT, oxymatrine; CIA, collagen-induced arthritis; DXM, dexamethasone; TNF- $\alpha$, tumor necrosis factor- $\alpha$; IL-17A, interleukin-17A.

scoring the clinical disease activity daily following secondary immunization. The arthritic scores reached a peak on day 25 . The OMT-treated groups had significantly decreased arthritic scores, compared with the model group between days 17 and 41 . The same efficacy of DXM (2 mg/kg) was observed (Fig. 2).

OMT reduces synovial inflammation and inflammatory articular destruction in SD rats with CIA. To investigate the inhibitory effects of OMT on arthritic activity in rats with CIA, histopathological assessment of the knee joints was performed using H\&E staining. Compared with the normal group (Fig. 3A), the knee joints of the model group rats revealed marked synovial hyperplasia and inflammatory cell infiltration into the joint capacity (Fig. 3B). The data of the DXM group showed a prominent reduction in synovial hyperplasia and inflammatory cell infiltration, compared with the model group (Fig. 3C). The CIA rats treated with 100 or $50 \mathrm{mg} / \mathrm{kg}$ OMT had lower levels of inflammatory cells infiltration, well-preserved joint spaces and minimal synovia hyperplasia (Fig. 3D-F). These results suggested that OMT inhibited synovial inflammation and inflammatory articular destruction at the knee joints in rats with CIA.

OMT suppresses inflammatory cytokine production. It is known that numerous cytokines are fundamental to the processes causing inflammation, articular destruction and the co-morbidities associated with RA in the joints of patients with RA. To investigate the mechanisms mediating

the decreased severity of CIA following OMT treatment, the present study evaluated the effect of OMT on the production of mediators of inflammation, which decreased the incidence and severity of CIA. The levels of TNF- $\alpha$ and IL-17A inflammatory cytokines in the serum drawn from the heart of CIA rats were measured using ELISA kits. The concentrations of total TNF- $\alpha$ and IL-17A in the serum of the CIA model group were significantly higher compared with the normal group $(\mathrm{P}<0.05)$, whereas OMT $(100,50$ and $25 \mathrm{mg} / \mathrm{kg})$ treatment of CIA rats markedly reduced the production of TNF- $\alpha$ and IL-17A inflammatory cytokines in the serum of the CIA rats (Fig. 4A and B). In addition, dose of $100 \mathrm{mg} / \mathrm{kg}$ OMT significantly inhibited the production of TNF- $\alpha$ and IL-17A, compared with a dose of $25 \mathrm{mg} / \mathrm{kg}(\mathrm{P}<0.05)$. The inhibitory effect on TNF- $\alpha$ and IL-17A levels in the serum of the CIA rats was higher in the OMT-treated groups, compared with those in the DXM-treated group $(\mathrm{P}<0.05)$. These data suggested that the administration of OMT may deactivate the inflammatory response of infiltrating and proliferating synovial cells in a dose-dependent manner.

OMT increases the expression of FOXP3 and decreases the expression of ROR $\mathrm{rt}$ in rat splenocytes. Total RNA was isolated from the spleen lymphocyte cells of either the OMT-treated CIA rats or CIA model rats, and the mRNA expression of Treg and Th17 cell-associated markers were examined using RT-qPCR analysis. The results showed that the mRNA level of FOXP3, a Treg cell-related molecule, was significantly upregulated in the spleen lymphocyte cells of the 


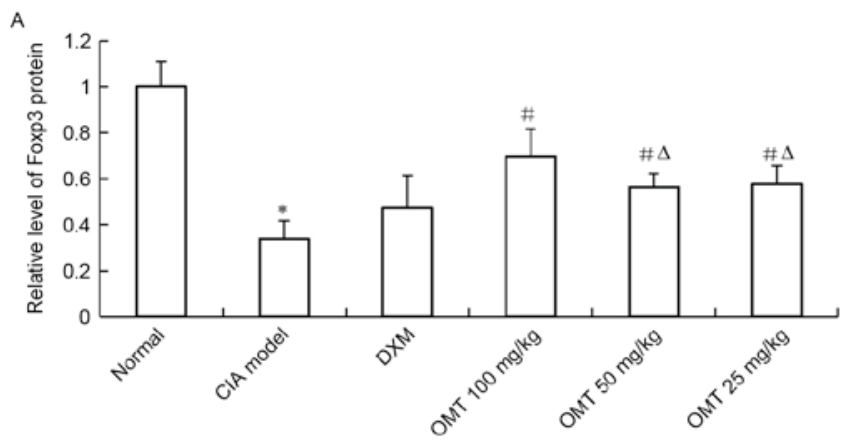

B

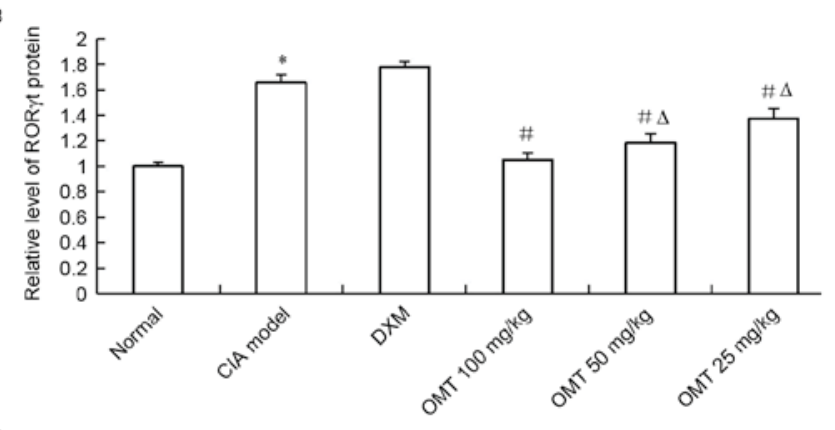

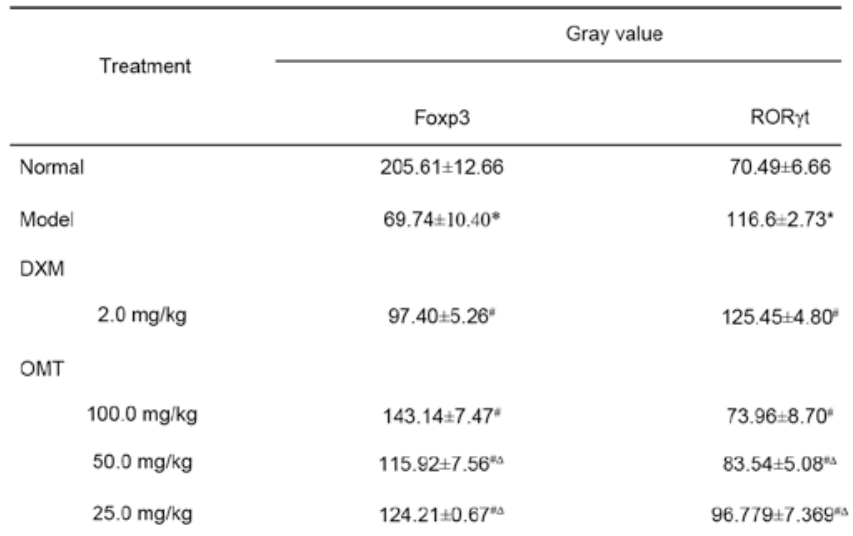

Figure 6. OMT increases the protein expression level of Foxp3 and decreases the protein expression level of ROR $\gamma$ t in rat splenocytes, detected using western blot analysis. (A) Foxp3. (B) ROR $\gamma$ t. (C) Gray values. ${ }^{*} \mathrm{P}<0.05$, compared with the normal group; ${ }^{*} \mathrm{P}<0.05$, compared with the CIA mode group; ${ }^{\Delta} \mathrm{P}<0.05$, compared with the $100 \mathrm{mg} / \mathrm{kg}$ group. OMT, oxymatrine; CIA, collagen-induced arthritis; DXM, dexamethasone; Foxp3, forkhead box P3; ROR $\gamma \mathrm{t}$, retinoic acid-related orphan receptor $\gamma \mathrm{t}$.

OMT-treated rats (Fig. 5A). By contrast, the mRNA level of the Th17 cell-associated molecule, ROR $\gamma$ t, was decreased in the OMT-treated CIA rats (Fig. 5B). The results of the western blot analysis also demonstrated that OMT treatment significantly suppressed the protein level of ROR $\gamma t$ and significantly increased that of FOXP3 in the rats' spleen lymphocytes (Fig. 6A-C). Thus, the administration of OMT in SD rats may ameliorate CIA by regulating the imbalance between Treg cells and Th17 cells.

\section{Discussion}

RA is an autoimmune disease, which can cause chronic joint inflammation and disability (39). The common treatment of such diseases is typically with immunosuppressants, which inhibit the immune response. However, the long-term use of immunosuppressive medications can have harmful side effects $(40,41)$. Therefore, novel drugs with high efficacy and low toxicity are urgently required. OMT is an alkaloid obtained from the traditional Chinese medicine, Sophora flavescens Ait, which has been reported to benefit patients suffering from inflammatory diseases, cancer and chronic hepatitis B (42). However, mechanistic evidence of the effect of OMT on the immune-inflammatory response in RA remains limited. In the present study, the potential therapeutic function of OMT in the CIA animal model was investigated. Paw swelling and arthritic scores are indices for measuring the anti-arthritic activity of various drugs, and were used in the present study to determine the activity of OMT at concentrations of 100,50 and $25 \mathrm{mg} / \mathrm{kg} / \mathrm{d} / \mathrm{i} . \mathrm{p}$. The results showed that rats in the OMT-administered groups had a significant reduction in paw volume and marked decrease in arthritic scores, compared with those in the CIA model group. In addition, histopathologic assessment of the joints of the OMT-treated rats revealed that OMT reduced synovial inflammation and inflammatory articular destruction, and ameliorated symptoms in the affected knee joint.

The most meaningful observation of the present study was that OMT inhibited the level of IL-17A induced by the inflammatory response, and upregulated Treg cells. It has been reported that the balance between Th17 and Treg cells has a significant role in the induction and progression of RA (43). Th17 cells, a novel $\mathrm{CD}^{+}{ }^{+}$Th cell subtype, produce cytokine profiles, including IL-17, IL-21, IL-22, IL-6 and TNF- $\alpha$. The IL-17 cytokine family includes six members: IL-17A, B, C, D, $\mathrm{E}$ (IL-25) and F $(44,45)$. It is well-documented that IL-17A is vital in the additive/synergistic effects induced with TNF- $\alpha$ and IL-1, two key pro-inflammatory cytokines in destructive arthritis, whereas Treg cells expressing FOXP3 possess anti-inflammatory activity (5-47). The significant suppression of pro-inflammatory cytokines, including IL-17A and TNF- $\alpha$, was examined in the spleen of OMT-treated CIA rats. As the reduction in the expression of IL-17 and Th17 cells, and the upregulation of Treg cells are significant properties in inhibiting inflammation, the results of the present study revealed that OMT possessed an anti-inflammatory function in autoimmune arthritis. ROR $\gamma \mathrm{t}$, lineage-specific transcription factors are essential for Th17 cell differentiation and the expression of IL-17. Thus, the inhibition of Th17 differentiation is therapeutically valuable, with suppression of differentiation and reduced production of Th17-associated pro-inflammatory cytokines. OMT markedly increased the expression of FOXP3 and suppressed the expression of ROR $\gamma \mathrm{t}$ in the rat splenocytes.

In previous years, substantial evidence has supported that an imbalance between Treg and Th17 cells is crucial in the immunopathogenesis of RA $(48,49)$. The differentiation of Th17 cells was induced by the activation of signal transducer and activator of transcription (STAT)3, however, the activation of STAT5 promoted the expression of FOXP3 in Treg cells (50). It has been reported that the transcription of IL-17 is regulated by the competitive binding of phosphorylated (p)STAT3 and pSTAT5 (51). Thus, pSTAT5 is a critical transcriptional factor for FOXP3 in regulating the differentiation of Treg cells. In the present study, OMT treatment enhanced the differentiation of Treg cells and inhibited Th17 cells. Information on the control of Th17 and Treg cells by OMT is limited. The results of the present study showed the 
possibility of OMT regulating the Treg/Th17 balance, which appears to be caused by the downregulation of $\mathrm{T}$ cell transcriptional regulators, including ROR $\gamma \mathrm{t}$, and the upregulation of FOXP3. Therefore, further investigations are required to clarify the effects of OMT on modulating Janus kinase-STAT signaling.

In conclusion, the present study investigated OMT as an immunomodulatory agent, involved in attenuating the inflammatory response at multiple levels, and suppressing synovial inflammation and cartilage destruction in CIA rats. These results assist in further elucidating the pathogenesis of immune-mediated inflammatory diseases, including RA. In addition, OMT may be a potent strategy for the treatment or prevention of RA.

\section{Acknowledgements}

The authors would like to thank all members of the Immunology Laboratory (Ningxia Medical University, Yinchuan, China) for their assistance. The present study was supported by the National Natural Science Foundation of China (grant no. 81560701) and the Ningxia Natural Science Foundation Program (grant nos. NZ14087 and NZ14139).

\section{References}

1. Helmick CG, Felson DT, Lawrence RC, Gabriel S, Hirsch R, Kwoh CK, Liang MH, Kremers HM, Mayes MD, Merkel PA, et al: Estimates of the prevalence of arthritis and other rheumatic conditions in the United States. Part I. Arthritis Rheum 58: $15-25,2008$.

2. Innala L, Sjöberg C, Möller B, Ljung L, Smedby T, Södergren A, Magnusson S, Rantapää-Dahlqvist S and Wållberg-Jonsson S: Co-morbidity in patients with early rheumatoid arthritis-inflammation matters. Arthritis Res Ther 18: 33, 2016.

3. Shegarfi H, Naddafi F and Mirshafiey A: Natural killer cells and their role in rheumatoid arthritis: Friend or foe? ScientificWorld Journal 2012: 491974, 2012.

4. MacNaul KL, Hutchinson NI, Parsons JN, Bayne EK and Tocci MJ: Analysis of IL-1 and TNF-alpha gene expression in human rheumatoid synoviocytes and normal monocytes by in situ hybridization. J Immunol 45: 4154-4166, 1990.

5. Azizi G, Jadidi-Niaragh F and Mirshafiey A: Th17 Cells in Immunopathogenesis and treatment of rheumatoid arthritis. Int J Rheum Dis 16: 243-253, 2013.

6. Zhu J, Yamane H and Paul WE: Differentiation of effector CD4 T cell populations (*). Annu Rev Immunol 28: 445-489, 2010.

7. Harrington LE, Hatton RD, Mangan PR, Turner H, Murphy TL, Murphy KM and Weaver CT: Interleukin 17-producing CD4+ effector $\mathrm{T}$ cells develop via a lineage distinct from the T helper type 1 and 2 lineages. Nat Immunol 6: 1123-1132, 2005.

8. Park H, Li Z, Yang XO, Chang SH, Nurieva R, Wang YH, Wang Y, Hood L, Zhu Z, Tian Q and Dong C: A distinct lineage of CD4 T cells regulates tissue inflammation by producing interleukin 17. Nat Immunol 6: 1133-1141, 2005.

9. Firestein GS: Evolving concepts of rheumatoid arthritis. Nature 423: 356-361, 2003.

10. Aarvak T, Chabaud M, Källberg E, Miossec P and Natvig JB Change in the Th1/Th2 phenotype of memory T-cell clones from rheumatoid arthritis synovium. Scand J Immunol 50: 1-9, 1999.

11. Talaat RM, Mohamed SF, Bassyouni IH and Raouf AA: Th1/Th2/Th17/Treg cytokine imbalance in systemic lupus erythematosus (SLE) patients: Correlation with disease activity. Cytokine 72: 146-153, 2015.

12. Heo YJ, Joo YB, Oh HJ, Park MK, Heo YM, Cho ML, Kwok SK, Ju JH, Park KS, Cho SG, et al: IL-10 suppresses Th17 cells and promotes regulatory $\mathrm{T}$ cells in the $\mathrm{CD} 4+\mathrm{T}$ cell population of rheumatoid arthritis patients. Immunol Lett 127: 150-156, 2010.

13. Louten J, Boniface K and de Waal Malefyt R: Development and function of TH17 cells in health and disease. J Allergy Clin Immunol 123: 1004-1011, 2009.
14. Dejaco C, Duftner C, Klauser A and Schirmer M: Altered T-cell subtypes in spondyloarthritis, rheumatoid arthritis and polymyalgia rheumatica. Rheumatol Int 30: 297-303, 2010.

15. Komatsu $\mathrm{N}$ and Takayanagi H: Regulatory $\mathrm{T}$ cells in Arthritis. Prog Mol Biol Transl Sci 136: 207-215, 2015.

16. Gizinski AM and Fox DA: T cell subsets and their role in the pathogenesis of rheumatic disease. Curr Opin Rheumatol 26: 204-210, 2014.

17. Alunno A, Manetti M, Caterbi S, Ibba-Manneschi L, Bistoni O, Bartoloni E, Valentini V, Terenzi R and Gerli R: Altered Immunoregulation in Rheumatoid Arthritis: The Role of Regulatory T Cells and Proinflammatory Th17 Cells and Therapeutic Implications. Mediators Inflamm 2015: 751793, 2015.

18. Nam JL, Winthrop KL, van Vollenhoven RF, Pavelka K, Valesini G, Hensor EM, Worthy G, Landewé R, Smolen JS, Emery P and Buch MH: Current evidence for the management of rheumatoid arthritis with biological disease-modifying antirheumatic drugs: A systematic literature review informing the EULAR recommendations for the management of RA. Ann Rheum Dis 69: 976-986, 2010.

19. Romas E, Gillespie MT and Martin TJ: Involvement of receptor activator of NFkappaB ligand and tumor necrosis factor-alpha in bone destruction in rheumatoid arthritis. Bone 30: 340-346, 2002.

20. Chan FK: The David Y. Graham lecture: Use of nonsteroidal antiinflammatory drugs in a COX-2 restricted environment. Am J Gastroenterol 103: 221-227, 2008.

21. Mosleh W and Farkouh ME: Balancing cardiovascular and gastrointestinal risks in patients with osteoarthritis receiving nonsteroidal anti-inflammatory drugs. A summary of guidelines from an international expert group. Pol Arch Med Wewn 126: $68-75,2016$.

22. Pope JE, Anderson JJ and Felson DT: A meta-analysis of the effects of nonsteroidal anti-inflammatory drugs on blood pressure. Arch Intern Med 153: 477-484, 1993.

23. McGettigan P and Henry D: Cardiovascular risk with non-steroidal anti-inflammatory drugs: Systematic review of population-based controlled observational studies. PLoS Med 8: e1001098, 2011.

24. Harrold LR, Reed GW, Harrington JT, Barr CJ, Saunders KC, Gibofsky A, Greenberg JD, John A, Devenport J and Kremer JM: The rheumatoid arthritis treat-to-target trial: A cluster randomized trial within the Corrona rheumatology network. BMC Musculoskelet Disord 15: 389, 2014

25. Smolen JS and Steiner G: Therapeutic strategies for rheumatoid arthritis. Nat Rev Drug Discov 2: 473-488, 2003.

26. Olsen NJ and Stein CM: New drugs for rheumatoid arthritis. N Engl J Med 350: 2167-2179, 2004.

27. Lee JD, Huh JE, Baek YH, Cho KC, Choi DY and Park DS: The Efficacy and Mechanism Action of RvCSd, a New herbal agent, on immune suppression and cartilage protection in a mouse model of rheumatoid arthritis. J Pharmacol Sci 109: 211-221, 2009.

28. Guzman JR, Koo JS, Goldsmith JR, Mühlbauer M, Narula A and Jobin C: Oxymatrine prevents NF- $\kappa \mathrm{B}$ nuclear translocation and ameliorates acute intestinal inflammation. Sci Rep 3: 1629, 2013.

29. Dong XQ, Yu WH, Hu YY, Zhang ZY and Huang M: Oxymatrine reduces neuronal cell apoptosis by inhibiting Toll-like receptor 4/nuclear factor kappa-B-dependent inflammatory responses in traumatic rat brain injury. Inflamm Res 60: 533-539, 2011.

30. Jiang G, Liu X, Wang M, Chen H, Chen Z and Qiu T: Oxymatrine ameliorates renal ischemia-reperfusion injury from oxidative stress through Nrf2/HO-1 pathway. Acta Cir Bras 30: 422-429, 2015.

31. Yao $\mathrm{N}$ and Wang $\mathrm{X}$ : In vitro immunomodulatory activity of oxymatrine on Toll-like receptor 9 signal pathway in chronic hepatitis B. Am J Chin Med 42: 1399-1410, 2014.

32. Li J, Jiang K and Zhao F: Oxymatrine suppresses proliferation and facilitates apoptosis of human ovarian cancer cells through upregulating microRNA-29b and downregulating matrix metalloproteinase-2 expression. Mol Med Rep 12: 5369-5374, 2015.

33. Wang W, Pei X, Xu M, Sun S, Zhang C, Mu K and Liu Z: The Protective Effect of Sodium Ferulate and Oxymatrine Combination on Paraquat-induced Lung Injury. Iran J Pharm Res 14: 573-583, 2015.

34. Wu C, Huang W, Guo Y, Xia P, Sun X, Pan X and Hu W: Oxymatrine inhibits the proliferation of prostate cancer cells in vitro and in vivo. Mol Med Rep 11: 4129-4134, 2015. 
35. Parvez MK, Arbab AH, Al-Dosari MS and Al-Rehaily AJ: Antiviral natural products against chronic hepatitis B: Recent Developments. Curr Pharm Des 22: 286-293, 2016.

36. Mason TJ and Matthews M: Aquatic environment, housing and management in the eighth edition of the guide for the care and use of laboratory animals: Additional considerations and recommendations. J Am Assoc Lab Anim Sci 51: 329-332, 2012.

37. Pfaffl MW: A new mathematical model for relative quantification in real-time RT-PCR. Nucleic Acids Res 29: e45, 2001.

38. Livak KJ and Schmittgen TD: Analysis of relative gene expression data using real-time quantitative PCR and the 2(-Delta Delta C(T)) method. Methods 25: 402-408, 2001.

39. Klarenbeek NB, Kerstens PJ, Huizinga TW, Dijkmans BA and Allaart CF: Recent advances in the management of rheumatoid arthritis. Br Med J 341: c6942, 2010.

40. Katsuragi T, Iwashige A and Tsukada J: Immunodeficiencyassociated Burkitt lymphoma developed in a patient receiving a long-term methotrexate therapy for rheumatoid arthritis. Rinsho Ketsueki 57: 9-14, 2016 (In Japanese).

41. Scott FI, Mamtani R, Brensinger CM, Haynes $\mathrm{K}$, Chiesa-Fuxench ZC, Zhang J, Chen L, Xie F, Yun H, Osterman MT, et al: Risk of nonmelanoma skin cancer associated with the use of immunosuppressant and biologic agents in patients with a history of autoimmune disease and nonmelanoma skin cancer. JAMA Dermatol 152: 164-172, 2016.

42. Wang X, Liu C, Wang J, Fan Y, Wang Z and Wang Y: Oxymatrine inhibits the migration of human colorectal carcinoma RKO cells via inhibition of PAI- 1 and the TGF- $\beta 1 /$ Smad signaling pathway. Oncol Rep 37: 747-753, 2017.

43. Estrada-Capetillo L, Hernández-Castro B, Monsiváis-Urenda A, Alvarez-Quiroga C, Layseca-Espinosa E, Abud-Mendoza C, Baranda L, Urzainqui A, Sánchez-Madrid F and González-Amaro R: Induction of Th17 lymphocytes and Treg cells by monocyte-derived dendritic cells in patients with rheumatoid arthritis and systemic lupus erythematosus. Clin Dev Immunol 2013: 584303, 2013.
44. Bettelli E, Korn T and Kuchroo VK: Th17: The third member of the effector T cell trlogy. Curr Opin Immunol 19: 652-657, 2007.

45. Weaver CT, Harrington LE, Mangan PR, Gavrieli M and Murphy KM: Th17: An effector CD4 T cell lineage with regulatory T Cell ties. Immunity 24: 677-688, 2006.

46. Komatsu N and Takayanagi H: Regulatory T cells in Arthritis. Prog Mol Biol Transl Sci 136: 207-215, 2015.

47. An Haack I, Derkow K, Riehn M, Rentinck MN, Kühl AA, Lehnardt S and Schott E: The Role of Regulatory CD4 T Cells in Maintaining Tolerance in a Mouse Model of autoimmune hepatitis. PLoS One 10: e0143715, 2015.

48. Lina C, Conghua W, Nan L and Ping Z: Combined treatment of etanercept and MTX reverses Th1/Th2, Th17/Treg imbalance in patients with rheumatoid arthritis. J Clin Immunol 31: 596-605, 2011.

49. Venkatesha SH, Dudics S, Weingartner E, So EC, Pedra J and Moudgil KD: Altered Th17/Treg balance and dysregulated IL-1 $\beta$ response influence susceptibility/resistance to experimental autoimmune arthritis. Int J Immunopathol Pharmacol 28: 318-328, 2015.

50. Fischer A: Human immunodeficiency: Connecting STAT3, Th17 and human mucosal immunity. Immunol Cell Biol 86: 549-551, 2008.

51. Yang XP, Ghoreschi K, Steward-Tharp SM, Rodriguez-Canales J, Zhu J, Grainger JR, Hirahara K, Sun HW, Wei L, Vahedi G, et al: Opposing regulation of the locus encoding IL-17 through direct, reciprocal actions of STAT3 and STAT5. Nat Immunol 12: 247-254, 2011. 